\title{
Important Note
}

Throughout this book, I will be drawing from the actual experiences of real individuals who have faced the issues and challenges involved in genetic testing. In order to protect the privacy of these generous individuals who were willing to allow us to look into their lives, I am not using their real names and I have changed details of their family histories and their stories. So if you think that you recognize someone, you will be mistaken.

The main goal of this book is to suggest a template or guide for decision making about genetic testing. There is no intention to endorse any of the specific decisions made by the individuals who have contributed to this study, whether those decisions were to have or not to have genetic testing, to share or not to share genetic information, or to choose or to reject a particular form of treatment or a particular means of prevention. Each of us must make our own decision, in the context of our own life, with input from qualified medical professionals. 

To Test or Not to Test 
